\title{
Gauss-Bonnet Braneworld Cosmology with Modified Induced Gravity on the Brane
}

\author{
Kourosh Nozari, ${ }^{1}$ Faeze Kiani, ${ }^{2}$ and Narges Rashidi ${ }^{2}$ \\ ${ }^{1}$ Center for Excellence in Astronomy and Astrophysics (CEAAI-RIAAM), P.O. Box 55134-441, Maragha, Iran \\ ${ }^{2}$ Department of Physics, Faculty of Basic Sciences, University of Mazandaran, P.O. Box 47416-95447, Babolsar, Iran \\ Correspondence should be addressed to Kourosh Nozari; knozari@umz.ac.ir
}

Received 21 May 2013; Accepted 19 August 2013

Academic Editor: Jose Edgar Madriz Aguilar

Copyright (c) 2013 Kourosh Nozari et al. This is an open access article distributed under the Creative Commons Attribution License, which permits unrestricted use, distribution, and reproduction in any medium, provided the original work is properly cited.

\begin{abstract}
We analyze the background cosmology for an extension of the DGP gravity with Gauss-Bonnet term in the bulk and $f(R)$ gravity on the brane. We investigate implications of this setup on the late-time cosmic history. Within a dynamical system approach, we study cosmological dynamics of this setup focusing on the role played by curvature effects. Finally, we constrain the parameters of the model by confrontation with recent observational data.
\end{abstract}

\section{Introduction}

One of the most significant astronomical observations in the last decade is the accelerated expansion of the universe [1-14]. One way to explain this accelerating phase of the universe expansion is invoking a dark energy component in the matter sector of the Einstein field equations [15-31]. However, it is possible also to modify the geometric part of the field equations to achieve this goal [32-45]. In the spirit of the second viewpoint, the braneworld model proposed by Dvali, Gabadadze, and Porrati (DGP) provides a natural explanation of late-time accelerated expansion in its selfaccelerating branch of the solutions [46-51]. Unfortunately, the self-accelerating branch of this scenario suffers from ghost instabilities [52, 53], and, therefore, it is desirable to invoke other possibilities in this braneworld setup. An amazing feature of the DGP setup is that the normal branch of this scenario, which is not self-accelerating, has the capability to realize phantom-like behavior without introducing any phantom field neither in the bulk nor on the brane [54-60]. By the phantom-like behavior one means that an effective energy density, which is positive, grows with time and its equation of state parameter $\left(\omega_{\text {eff }}=p_{\text {eff }} / \rho_{\text {eff }}\right)$ stays always less than -1 . The phantom-like prescription breaks down if this effective energy density becomes negative. An interesting extension of the DGP setup is possible modification of the induced gravity on the brane (We call the $f(R)$ term on the brane the modified induced gravity since this braneworld scenario is an extension of the DGP model. In the DGP model, the gravity is induced on the brane through interaction of the bulk graviton with loops of matter on the brane. So the phrase "induced gravity" is coming from the DGP character of our model.) on the brane. This can be achieved by treating the induced gravity in the framework of $f(R)$ gravity [61-66]. This extension can be considered as a manifestation of the scalar-tensor gravity on the brane since $f(R)$ gravity can be reconstructed as a general relativity plus a scalar field [32-45]. Some features of this extension such as self-acceleration in the normal branch of the scenario are studied recently [61-68]. Here, we generalize this viewpoint to the case that the Gauss-Bonnet curvature effect is also taken into account. We consider a DGP-inspired braneworld model where the induced gravity on the brane is modified in the spirit of $f(R)$ gravity, and the bulk action contains the Gauss-Bonnet term to incorporate higher-order curvature effects. Our motivation is to study possible influences of the curvature corrections on the cosmological dynamics on the normal branch of the DGP setup. We analyze the background cosmology and possible realization of the phantom-like behavior in this setup. By introducing a curvature fluid that plays the role of dark energy component, we show that this model realizes phantom-like behavior on the normal branch 
of the scenario in some subspaces of the model parameter space, without appealing to phantom fields neither in the bulk nor on the brane and by respecting the null energy condition in the phantom-like phase of expansion. We show also that in this setup there is smooth crossing of the phantom divide line by the equation of state parameter and the universe transits smoothly from a quintessence-like phase to a phantom-like phase. We present a detailed analysis of cosmological dynamics in this setup within a dynamical system approach in order to reveal some yet unknown features of these kinds of models in their phase space. Finally, confrontation of our model with recent observational data leads us to some constraint on model parameters.

\section{The Setup}

2.1. Gauss-Bonnet Braneworld with Induced Gravity on the Brane. The action of a GBIG (the Gauss-Bonnet term in the bulk and induced gravity on the brane) braneworld scenario can be written as follows [69-80]:

$$
S=S_{\text {bulk }}+S_{\text {brane }}
$$

where, by definition,

$$
S_{\text {bulk }}=\frac{1}{16 \pi G^{(5)}} \int d^{5} x \sqrt{-g}\left[\mathscr{R}^{(5)}-2 \Lambda^{(5)}+\alpha \mathscr{L}_{\mathrm{GB}}\right],
$$

with

$$
\begin{gathered}
\mathscr{L}_{\mathrm{GB}}=\left(\mathscr{R}^{(5)}\right)^{2}-4 \mathscr{R}_{\mathrm{AB}}^{(5)} \mathscr{R}^{(5) \mathrm{AB}}+\mathscr{R}_{\mathrm{ABCD}}^{(5)} \mathscr{R}^{(5) \mathrm{ABCD}}, \\
S_{\text {brane }}=\frac{1}{16 \pi G^{(4)}} \int d^{4} x \sqrt{-q}\left[\mathscr{R}-2 \Lambda^{(4)}\right] .
\end{gathered}
$$

$G^{(5)}$ is the 5D Newton constant in the bulk, and $G^{(4)}$ is the corresponding $4 \mathrm{D}$ quantity on the brane. $\mathscr{L}_{\mathrm{GB}}$ is the Gauss-Bonnet term, and $\alpha$ is a constant with dimension of $[\text { length }]^{2} . q$ is the induced metric on the brane. We choose the coordinate of the extra dimension to be $y$, so that the brane is located at $y=0$. The DGP crossover distance, which is defined as

$$
r_{c}=\frac{G^{(5)}}{G^{(4)}}=\frac{\kappa_{5}^{2}}{2 \kappa_{4}^{2}},
$$

has the dimension of [length] and will appear in our forthcoming equations. We note that this scenario is UV/IR complete in some sense, since it contains both the GaussBonnet term as a string-inspired modification of the UV (ultraviolet) sector and the induced gravity as IR (infrared) modification to the general relativity. The cosmological dynamics of this GBIG scenario is described fully by the following Friedmann equation [81-84]:

$$
\begin{aligned}
& {\left[H^{2}+\frac{k}{a^{2}}-\frac{8 \pi G^{(4)}(\rho+\lambda)}{3}\right]^{2}} \\
& \quad=\frac{4}{r_{c}^{2}}\left[1+\frac{8 \alpha}{3}\left(H^{2}+\frac{k}{a^{2}}+\frac{U}{2}\right)\right]^{2}\left(H^{2}+\frac{k}{a^{2}}-U\right),
\end{aligned}
$$

where

$$
\begin{gathered}
U=-\frac{1}{4 \alpha} \pm \frac{1}{4 \alpha} \sqrt{1+4 \alpha\left(\frac{\Lambda^{(5)}}{6}+\frac{2 \mathscr{E}_{0} G^{(5)}}{a^{4}}\right)}, \\
\lambda \equiv \frac{\Lambda^{(4)}}{8 \pi G^{(4)}} .
\end{gathered}
$$

$\mathscr{E}_{0}$ is referred to hypothetically as the mass of the bulk black hole, and the corresponding term is called the bulk radiation term. Note that, when one adopts the positive sign, the above equation can be reduced to the generalized DGP model as $\alpha \rightarrow 0$, but the branch with negative sign cannot be reduced to the generalized DGP model in this regime. Therefore, we just consider the plus sign of the above equation [81-84]. We note that, depending on the choice of the bulk space, the brane FRW equations are different (see [85] for details). The bulk space in the present model is a 5-dimensional AdS black hole. In which follows, we assume that there is no cosmological constant on the brane or in the bulk; that is, $\Lambda^{(4)}=\Lambda^{(5)}=0$. Also, we ignore the bulk radiation term since it decays very fast in the early stages of the evolution (note, however, that this term is important when one treats cosmological perturbations on the brane). So the Friedmann equation in this case reduces to the following form:

$$
\left[H^{2}-\frac{8 \pi G^{(4)}}{3} \rho\right]^{2}=\frac{4}{r_{c}^{2}}\left[1+\frac{8 \alpha}{3} H^{2}\right]^{2} H^{2} .
$$

It has been shown that it is possible to realize the phantomlike behavior in this setup without introducing any phantom matter on the brane [86-91]. In which follows, we generalize this setup to the case that induced gravity on the brane is modified in the spirit of $f(R)$ gravity, and we explore the cosmological dynamics of this extended braneworld scenario.

2.2. Modified GBIG Gravity. In this subsection, we firstly formulate a GBIG scenario that induced gravity on the brane acquires a modification in the spirit of $f(R)$ gravity. To obtain the generalized Friedmann equation of this model, we proceed as follows. Firstly, the Friedmann equation for pure DGP scenario is given as follows [49-51, 92, 93]:

$$
\begin{aligned}
\epsilon \sqrt{H^{2}}-\frac{\mathscr{E}_{0}}{a^{4}}-\frac{\Lambda_{5}}{6}+\frac{k}{a^{2}} \\
\quad=r_{c}\left[\left(H^{2}+\frac{k}{a^{2}}\right)-\frac{8 \pi G^{(4)}}{3}(\rho+\lambda)\right],
\end{aligned}
$$

where $\epsilon= \pm 1$ is corresponding to two possible embeddings of the brane in the bulk. Considering a Minkowski bulk with $\Lambda_{5}=0$ and by setting $\mathscr{E}_{0}=0$ with a tensionless brane $(\lambda=0)$, for a flat brane $(k=0)$, we find that

$$
H^{2}=\frac{8 \pi G^{(4)}}{3} \rho \pm \frac{H}{r_{c}} .
$$

The normal branch of the scenario is corresponding to the minus sign on the right-hand side of this equation. 
The second term on the right is the source of the phantomlike behavior on the normal branch: the key feature of this phase is that the brane is extrinsically curved in such a way that shortcuts through the bulk allow gravity to screen the effects of the brane energy-momentum contents at Hubble parameters $H \sim r_{c}^{-1}$, and this is not the case for the selfaccelerating phase [54-60].

In the next step, we incorporate possible modification of the induced gravity by inclusion of a $f(R)$ term on the brane. This extension can be considered as a manifestation of the scalar-tensor gravity on the brane. In this case, we find the following generalized Friedmann equation (see, e.g., [61-68, 92, 93]):

$$
\begin{aligned}
& \epsilon \sqrt{H^{2}-\frac{M G^{(5)}}{a^{4}}-\frac{\Lambda_{5}}{6}+\frac{k}{a^{2}}} \\
& =r_{c}\left[\left(H^{2}+\frac{k}{a^{2}}\right) f^{\prime}(R)-\frac{8 \pi G^{(4)}}{3}\right. \\
& \left.\quad \times\left[\rho+\lambda+\left(\frac{1}{2}\left[R f^{\prime}(R)-f^{\prime}(R)\right]-3 H \dot{R} f^{\prime \prime}(R)\right)\right]\right],
\end{aligned}
$$

where a prime marks derivative with respect to $R$. In the third step, we need the GBIG Friedmann equation in the absence of any modification of the induced gravity on the brane, that is, without $f(R)$ term on the brane. This has been obtained in the previous subsection; see (5). Now, we have all prerequisites to obtain the Friedmann equation of our GBIGmodified gravity scenario. The comparison between previous equations gives this Friedmann equation of cosmological dynamics as follows (we note that this equation can be derived using the generalized junction conditions on the brane straightforwardly; see [81-84]):

$$
H^{2}=\frac{\kappa_{4}^{2}}{3 f^{\prime}(R)} \rho+\frac{\kappa_{4}^{2}}{3} \rho_{\text {curv }} \pm \frac{1}{r_{c} f^{\prime}(R)}\left(1+\frac{8}{3} \alpha H^{2}\right) H,
$$

where we have defined hypothetically the following energy density corresponding to curvature effect:

$$
\rho_{\text {curv }}=\frac{1}{f^{\prime}(R)}\left(\frac{1}{2}\left[R f^{\prime}(R)-f(R)\right]-3 H \dot{R} f^{\prime \prime}(R)\right) .
$$

Note that, to obtain this relation, $\mathscr{C}_{0}, \Lambda_{5}, \lambda$, and $k$ are set equal to zero. From now on, we restrict our attention to the normal branch of the scenario, that is, the minus sign in (11) because there are no ghost instabilities in this branch only if the DGP character of the model is considered.

Note, however, that although we refer to the normal (ghost-free) branch of the DGP model (in the sense that for $f(R)=R$ the obtained solutions reduce to this branch) as an indication of the ghost-free property of the considered solutions, it is not a priori guaranteed that, on the obtained de Sitter backgrounds which generalize the normal DGP branch, the ghost does not reappear. In fact, the ghost on the self-accelerated branch of the DGP model is entirely the problem of the de Sitter background. Within the crossover scale $r_{c}$ which is the horizon for the self-accelerated branch, the theory reduces to a scalar-tensor model, with the scalar sector (brane bending mode) being described by a simple Galileon self-interaction [94] as

$$
\mathscr{L}_{\pi}=\pi \square \pi-\frac{(\partial \pi)^{2} \square \pi}{\Lambda^{3}},
$$

which, in spite of the presence of higher derivatives, propagates a single healthy degree of freedom. The ghost on the selfaccelerated branch arises merely due to the fact that $\pi$ gets a nontrivial profile and the kinetic term for its perturbation flips the sign on the background. A similar argument can be applied in the present work to overcome the ghost instabilities in this extended braneworld setup.

In which follows, we assume that the energy density $\rho$ on the brane is due to cold dark matter (CDM) with $\rho_{m}=$ $\rho_{0 m}(1+z)^{3}$. We can rewrite the Friedmann equation in terms of observational parameters such as the redshift $z$ and dimensionless energy densities $\Omega_{i}$ as follows:

$$
\begin{aligned}
E^{2}= & \frac{\Omega_{m}}{f^{\prime}(R)}(1+z)^{3}+\Omega_{\text {curv }}(1+z)^{3\left(1+w_{\text {curv }}\right)} \\
& -2 \frac{\sqrt{\Omega_{r_{c}}}}{f^{\prime}(R)}\left[1+\Omega_{\alpha} E^{2}(z)\right] E(z),
\end{aligned}
$$

where

$$
\begin{gathered}
E(z) \equiv \frac{H}{H_{0}}, \\
\Omega_{m} \equiv \frac{\kappa_{4}^{2}}{3 H_{0}^{2}} \rho_{0 m}, \quad \Omega_{\alpha} \equiv \frac{8}{3} \alpha H_{0}^{2}, \\
\Omega_{r_{c}} \equiv \frac{1}{4 r_{c}^{2} H_{0}^{2}}, \quad \Omega_{\text {curv }} \equiv \frac{\kappa_{4}^{2}}{3 H_{0}^{2}} \rho_{0 \text { curv }}, \\
w_{\text {curv }}=\frac{p_{\text {curv }}}{\rho_{\text {curv }}} .
\end{gathered}
$$

$p_{\text {curv }}$, the hypothetical pressure of the curvature effect, can be obtained by the following equation of continuity:

$$
\begin{aligned}
\dot{\rho}_{\text {curv }} & +3 H\left(\rho_{\text {curv }}+p_{\text {curv }}+\frac{\dot{R} f^{\prime \prime}(R)}{r_{c}\left[f^{\prime}(R)\right]^{2}}\right) \\
& =\frac{3 H_{0}^{2} \Omega_{m} \dot{R} f^{\prime \prime}(R)}{\left[f^{\prime}(R)\right]^{2}} a^{-3} .
\end{aligned}
$$

One can obtain a constraint on the cosmological parameters of the model at $z=0$ as follows:

$$
\Omega_{m}=1-\Omega_{\text {curv }}+2 \sqrt{\Omega_{r_{c}}}\left(1+\Omega_{\alpha}\right) .
$$

Note that we have used the normalization $\left.f^{\prime}(R)\right|_{z=0}=1$ in this relation which is observationally a viable assumption. 


\section{Cosmological Dynamics in the Modified GBIG Scenario}

Now, we study cosmological dynamics in this setup. To solve the Friedmann equation for the normal branch of this scenario, it is convenient (following the papers by BohamdiLopez in [86-90]) to introduce the dimensionless variables as follows:

$$
\begin{gathered}
\bar{H} \equiv \frac{8}{3} \frac{\alpha}{r_{c} f^{\prime}(R)} H=2 \Omega_{\alpha} \sqrt{\Omega_{r}} E(z), \\
\bar{\rho} \equiv \frac{32}{27} \frac{\kappa_{5}^{2} \alpha^{2}}{\left[r_{c} f^{\prime}(R)\right]^{3}}\left(\rho_{m}+f^{\prime}(R) \rho_{\text {curv }}\right) \\
=4 \Omega_{\alpha}^{2} \Omega_{r}\left[\Omega_{m}(1+z)^{3}+\Omega_{\text {curv }}(1+z)^{3\left(1+w_{\text {curv }}\right)}\right], \\
b \equiv \frac{8}{3} \frac{\alpha}{\left[r_{c} f^{\prime}(R)\right]^{2}}=4 \Omega_{\alpha} \Omega_{r} .
\end{gathered}
$$

An effective crossover distance which appeared on the righthand side of these relations can be defined as follows,

$$
r \equiv r_{c} f^{\prime}(R)
$$

and, by definition, $\Omega_{r} \equiv 1 / 4 r^{2} H_{0}^{2}$. Then, the Friedmann equation can be rewritten as

$$
\bar{H}^{3}+\bar{H}^{2}+b \bar{H}-\bar{\rho}=0 .
$$

The number of real roots of this equation is determined by the sign of the discriminant function $\mathcal{N}$ defined as

$$
\mathcal{N}=Q^{3}+S^{2},
$$

where $Q$ and $S$ are defined as

$$
\begin{gathered}
Q=\frac{1}{3}\left(b-\frac{1}{3}\right), \\
S=\frac{1}{6} b+\frac{1}{2} \bar{\rho}-\frac{1}{27},
\end{gathered}
$$

respectively. We can rewrite $\mathcal{N}$ as

$$
\mathcal{N}=\frac{1}{4}\left(\bar{\rho}-\bar{\rho}_{1}\right)\left(\bar{\rho}-\bar{\rho}_{2}\right)
$$

where

$$
\begin{aligned}
& \bar{\rho}_{1}=-\frac{1}{3}\left\{b-\frac{2}{9}\left[1+\sqrt{(1-3 b)^{3}}\right]\right\}, \\
& \bar{\rho}_{2}=-\frac{1}{3}\left\{b-\frac{2}{9}\left[1-\sqrt{(1-3 b)^{3}}\right]\right\} .
\end{aligned}
$$

In which follows, we consider just the real and positive roots of the Friedmann equation (20). For $0<b<1 / 4, \bar{\rho}_{1}>0$ and $\bar{\rho}_{2}<0$. Then, the number of real roots of the cubic Friedmann equation depends on the minimum energy density of the brane and the situation of $\rho_{1}$ relative to this minimum. Since, in our setup, curvature effect plays the role of the dark energy component on the brane, we can consider two different regimes to determine the minimum value of $\bar{\rho}$ as follows.
(1) $w_{\text {curv }}>-1$ : in this case, curvature fluid plays the role of quintessence component; then, the minimum value happens asymptotically at $z=-1$, and we will obtain $\bar{\rho}_{\text {min }}=0$. In this situation, we can define three possible regimes: a high-energy regime with $\bar{\rho}_{1}<\bar{\rho}$, a limiting regime with $\bar{\rho}_{1}=\bar{\rho}$, and a low-energy regime with $\bar{\rho}_{1}>\bar{\rho}$. In each of these cases, depending on the sign of $N$, there are different solutions [86-91].

(2) $w_{\text {curv }}<-1$ : in this case, the curvature fluid plays the role of a phantom component (we will investigate its phantom-like behavior in the next section), and the minimum value of $\bar{\rho}$ happens at $z=0.18$. So we find the $\bar{\rho}_{\min }$ as follows:

$$
\bar{\rho}_{\text {min }}=4 \Omega_{\alpha}^{2} \Omega_{r_{c}}\left[0.43+\Omega_{\text {curv }}(1+0.18)^{3\left(1+w_{\text {curv }}\right)}\right] \text {, }
$$

where we have set $\Omega_{m}=0.26$. We note that $w_{\text {curv }}$ is not constant, and, as we will show, it evolves from quintessence to phantom phase. We note also that the value of redshift when $\bar{\rho}_{\text {min }}$ occurs (i.e., $z=0.18$ ) has no dependence on the values of $w_{\text {curv }}$. Here, we treat only the case $\bar{\rho}_{1}<\bar{\rho}_{\text {min }}$ with details. When this condition is satisfied, the function $\mathcal{N}$ is positive, and there is a unique solution for expansion of the brane described by

$$
\bar{H}_{1}=\frac{1}{3}\left[2 \sqrt{1-3 b} \cosh \left(\frac{\eta}{3}\right)\right],
$$

where $\eta$ is defined as

$$
\cosh (\eta)=\frac{S}{\sqrt{-Q^{3}}} .
$$

We note that this condition provides a constraint on the dimensionless parameters $\Omega_{i}$ as follows:

$$
\begin{aligned}
- & \frac{1}{3 b}\left[b-\frac{2}{9}\left[1+\sqrt{(1-3 b)^{3}}\right]\right] \\
< & \Omega_{\alpha}\left[0.43+\Omega_{\text {curv }}(1+0.18)^{3\left(1+w_{\text {curv }}\right)}\right] .
\end{aligned}
$$

Figure 1 shows the phase space of the above relation. In this figure, we have defined $\Psi \equiv 0.43+\Omega_{\text {curv }}(1+0.18)^{3\left(1+w_{\text {curv }}\right)}$. The relation (28) is fulfilled for upper region (region II) of this figure. In this case, there are three possible regimes as was mentioned above. A point that should be emphasized here is the fact that, in the presence of modified induced gravity on the brane, the solution of the generalized Friedmann equation (11) is actually rather involved due to simultaneous presence of $H, \dot{H}$, and $\ddot{H}$. A thorough analysis of this problem is out of the scope of this study, but there are some attempts (such as cosmography) in this direction to construct an operational framework to treat this problem; see, for instance [41]. Here, we have tried to find a solution of (11) by using the discriminant function $\mathcal{N}$, the result of which is given by (26). However, we note that a complete analysis is needed, for instance, in the framework of cosmography of brane $f(R)$ gravity [95].

To investigate cosmology described by solution (26), we rewrite the original Friedmann equation in the following 


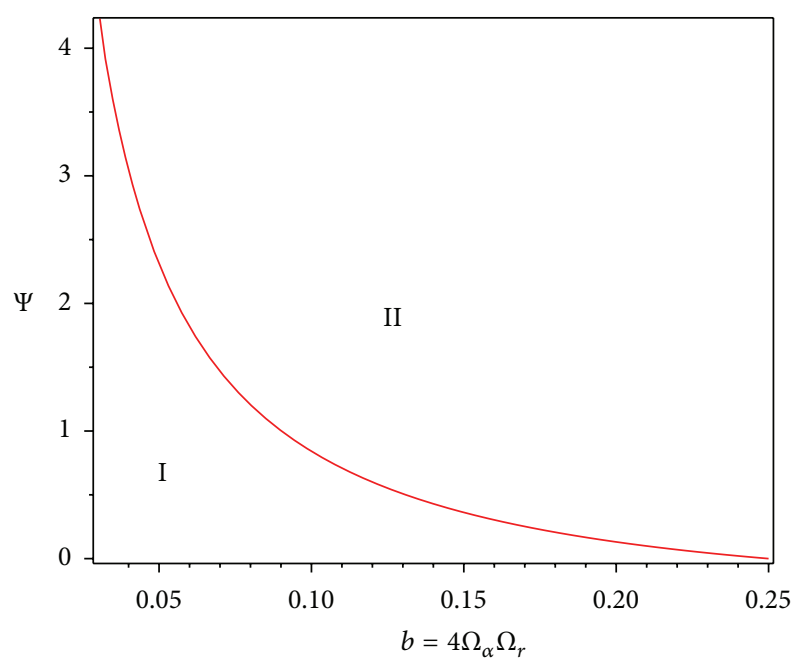

FIGURE 1: The upper region (region II) of this figure is corresponding to the set $\left(\Omega_{r}, \Omega_{\alpha}, \Omega_{\text {curv }}, w_{\text {curv }}\right)$ that fulfils inequality (28).

form in order to create a general relativistic description of our model:

$$
H^{2}=\frac{\left(\kappa_{4}^{2}\right)_{\mathrm{eff}}}{3} \rho_{m}+\rho_{\text {curv }}-\frac{H}{r_{c} f^{\prime}(R)}\left(1+\frac{8}{3} \alpha H^{2}\right),
$$

where $\rho_{\text {curv }}$ is defined in (12). Comparing this relation with the Friedmann equation in GR

$$
H^{2}=\frac{\kappa_{4}^{2}}{3}\left(\rho_{m}+\rho_{\mathrm{eff}}\right)
$$

we obtain an effective energy density

$$
\frac{\kappa_{4}^{2}}{3} \rho_{\mathrm{eff}}=\rho_{\text {curv }}-\frac{H}{r_{c} f^{\prime}(R)}\left(1+\frac{8}{3} \alpha H^{2}\right),
$$

which can be rewritten as follows:

$$
\rho_{\text {eff }}=\Omega_{\text {curv }}(1+z)^{3\left(1+w_{\text {curv }}\right)}-\frac{2 \sqrt{\Omega_{r_{c}}}}{f^{\prime}(R)}\left(1+\Omega_{\alpha} E^{2}\right) E(z) .
$$

The dependence of $\rho_{\text {eff }}$ on the redshift depends itself on the regimes introduced above and the form of $f(R)$ function. Figure 2 shows the variation of $\rho_{\text {eff }}$ versus the redshift for

$$
f(R)=R-(n-1) \zeta^{2}\left(\frac{R}{\zeta^{2}}\right)^{n}
$$

with $n=0.25$. This value of $n$ lies well in the range of observationally acceptable values of $n$ from solar-system tests [32-45]. (We note, however, that the key issue with regards to passing solar-system tests is not the value of $n$ but the value of $f^{\prime}(R)$ today. In fact, experimental data tell us that $f^{\prime}(R)-1<10^{-6}$, when $f^{\prime}(R)$ is parameterized to be exactly 1 in the far past.)

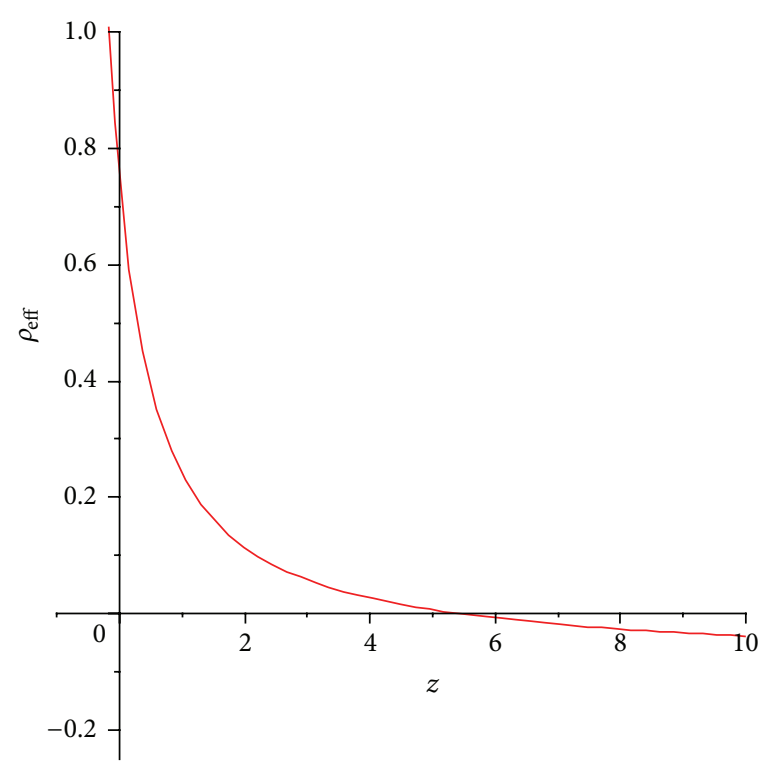

FIGURE 2: Variation of effective energy density versus the redshift for a specific $f(R)$ model described as (33) with $n=0.25$.

The effective energy density shows a phantom-like behavior; that is, it increases with cosmic time. This is a necessary condition to have phantom-like behavior, but it is not sufficient: we should check status of the deceleration parameter and also equation of state parameter. In a general relativistic description of our model, one can rewrite the energy conservation equation as follows:

$$
\dot{\rho}_{\text {eff }}+3 H\left(1+\omega_{\text {eff }}\right) \rho_{\text {eff }}=0
$$

which leads to the following relation for $1+\omega_{\text {eff }}$ :

$$
1+\omega_{\mathrm{eff}}=-\frac{\dot{\rho}_{\mathrm{eff}}}{3 H \rho_{\mathrm{eff}}} .
$$

Figure 3 shows variation of the effective equation of state parameter versus the redshift for a specific $f(R)$ model described as (33) with $n=0.25$. The effective equation of state parameter transits to the phantom phase $1+\omega_{\text {eff }}<0$, but there is no smooth crossing of the phantom divide line in this setup. We note that adopting other general ansatz, such as the Hu-Sawicki model [96]

$$
f(R)=R-R_{c} \frac{\alpha_{0}\left(R / R_{c}\right)^{n}}{1+\beta_{0}\left(R / R_{c}\right)^{n}}
$$

(where both $\alpha$ and $R_{c}$ are free positive parameters), does not change this result in our framework. The deceleration parameter defined as

$$
q \equiv-1-\frac{\dot{H}}{H^{2}}
$$




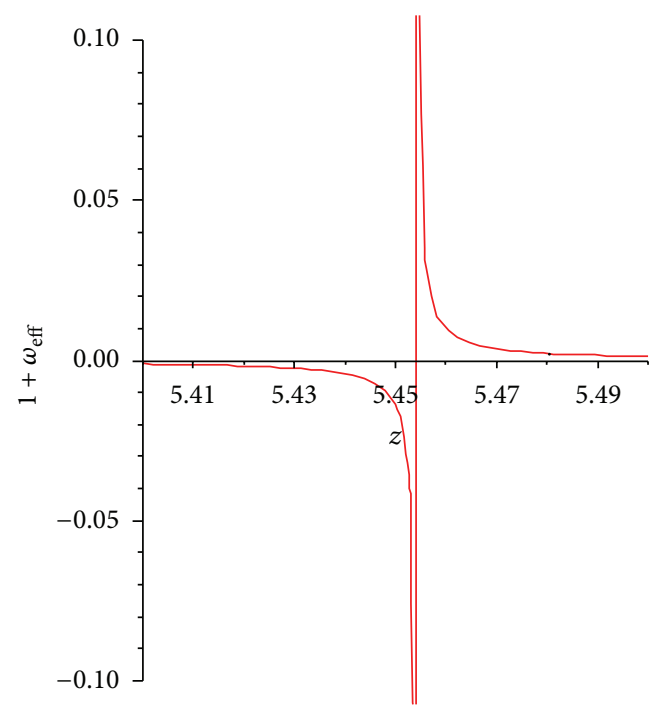

FIGURE 3: Variation of effective equation of state parameter versus the redshift for a specific $f(R)$ model described as (33) with $n=0.25$.

takes the following form in our setup:

$$
\begin{aligned}
q= & -1-\frac{\Omega_{m}(1+z)^{3}\left((3 / 2)-\left(\dot{f}^{\prime}(R) / f^{\prime}(R)\right)\left(H_{0} / E(z)\right)\right)}{f^{\prime}(R) E^{2}(z)+\sqrt{\Omega_{r_{c}}}\left(1+3 \Omega_{\alpha} E^{2}(z)\right) E(z)} \\
& -\left(\sqrt{\Omega_{r_{c}}} \frac{\dot{f}^{\prime}(R)}{f^{\prime}(R)}\left(1+\Omega_{\alpha} E^{2}(z)\right) \frac{1}{H_{0}}\right. \\
& \left.-\frac{3}{2} \Omega_{\text {curv }} f^{\prime}(R)(1+z)^{3\left(1+\omega_{c}\right)}\left(1+\omega_{c}\right)\right) \\
& \times\left(f^{\prime}(R) E^{2}(z)+\sqrt{\Omega_{r_{c}}}\left(1+3 \Omega_{\alpha} E^{2}(z)\right) E(z)\right)^{-1} .
\end{aligned}
$$

In this relation, $H$ and $f(R)$ are defined as (26) and (33). Figure 4 shows variation of $q$ versus the redshift for $n=0.25$. The universe enters the accelerated phase of expansion at $z \simeq 2$. Another important issue to be investigated in this setup is the big-rip singularity. To avoid super acceleration on the brane, it is necessary to show that Hubble rate decreases as the brane expands and that there is no big-rip singularity in the future. Figure 5 shows variation of $\dot{H}$ versus $z$. We see that in this model $\dot{H}<0$ always, and, therefore, there is no super-acceleration or future big-rip singularity in this setup. All of the previous considerations show that this model accounts for realization of the phantom-like behavior without introducing a phantom field neither on the brane nor in the bulk. Nevertheless, we have to check the status of the null energy condition in this setup. Figure 6 shows the variation of $(\rho+p)_{\text {tot }}$ versus the redshift. We see that this condition is fulfilled at least in some subspaces of the phantom-like region of the model parameter space.

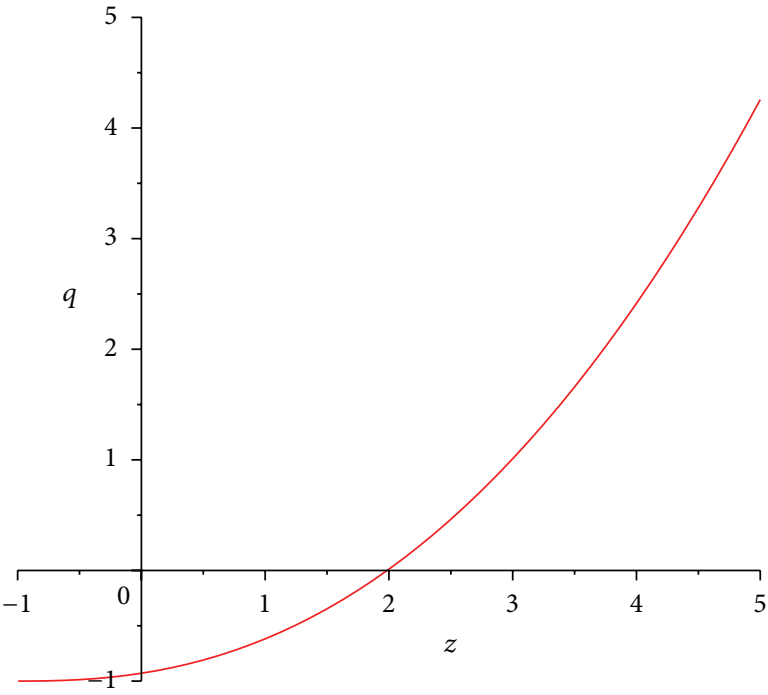

FIgURE 4: Variation of the deceleration parameter $q$ versus $z$ for $n=0.25$. The transition from deceleration to the acceleration phase occurs at $z \approx 2$.

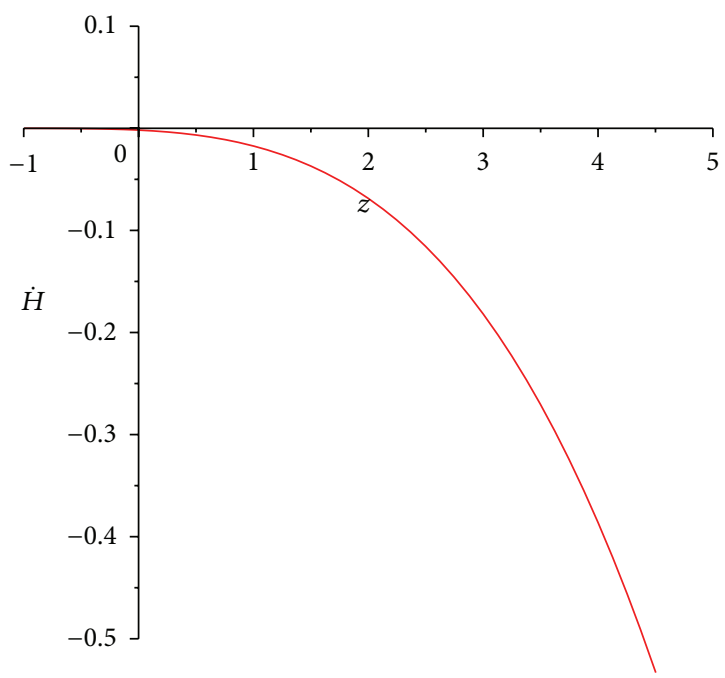

FIGURE 5: In this model, $\dot{H}$ is always negative, and, therefore, there is no superacceleration or big-rip singularity.

\section{A Dynamical System Viewpoint}

Up to this point, we have shown that there are effective quantities that create an effective phantom-like behavior on the brane. In this respect, one can define a potential related to the effective phantom scalar field $\phi_{\text {eff }}$ as follows [97]:

$$
\begin{gathered}
\frac{V_{\mathrm{eff}}(z)}{3 H_{0}^{2}}=E^{2}-\frac{\Omega_{m}(1+z)^{3}}{f^{\prime}(R)}+\frac{1}{2} \frac{d\left(E^{2}-\left(\Omega_{m}(1+z)^{3} / f^{\prime}(R)\right)\right)}{d \ln (1+z)^{3}}, \\
\frac{\phi_{\mathrm{eff}}(z)}{\sqrt{3}}=-\int \frac{d z}{(1+z) E} \sqrt{\frac{d\left(E^{2}-\left(\Omega_{m}(1+z)^{3} / f^{\prime}(R)\right)\right)}{d \ln (1+z)^{3}}} .
\end{gathered}
$$




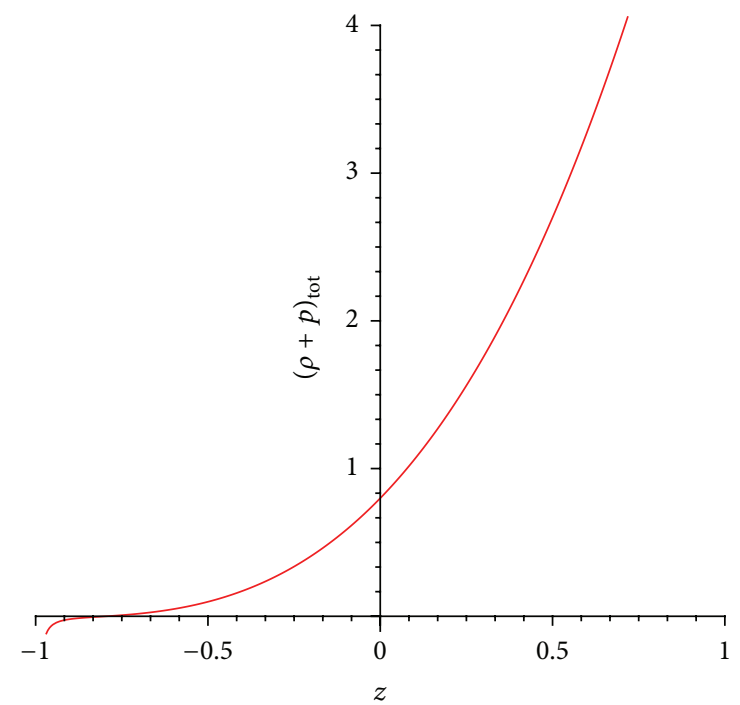

FIGURE 6: The status of the null energy condition in this model.

We note that in principle these equations can lead to $V_{\text {eff }}=$ $V_{\text {eff }}\left(\phi_{\text {eff }}\right)$, but in practice the inversion cannot be performed analytically. Now, we define the following normalized expansion variables [98-101]:

$$
\begin{gathered}
x=\frac{\sqrt{\Omega_{m}}}{a^{3 / 2} E}, \quad y=\frac{\sqrt{\Omega_{\text {curv }} f^{\prime}(R)}}{a^{(3 / 2)\left(1+w_{\text {curv }}\right)} E}, \\
v=\frac{\sqrt{\Omega_{r_{c}}}}{E}, \quad x=\sqrt{\Omega_{\alpha} E .}
\end{gathered}
$$

With these definitions, the Friedmann equation (14) takes the following form:

$$
x^{2}+y^{2}-2 v\left(1+\chi^{2}\right)=f^{\prime}(R) .
$$

This constraint means that the phase space can be defined by the relation $x^{2}+y^{2}-2 v \geq 1$, since $f^{\prime}(R)-1<10^{-6}$ by solarsystem constraints and $v$ is a positive quantity. Introducing the new time variable $\tau=\ln a$ and eliminating $\chi$ and $E$, we obtain the following autonomous system:

$$
\begin{gathered}
x^{\prime}=x\left(q-\frac{1}{2}\right), \\
y^{\prime}=\frac{y}{y^{2}-1}\left[\frac{3}{2}\left(1+w_{\text {curv }}\right)\left(1+x^{2}\right)-(q+1)\right. \\
\left.\times\left[1+x^{2}-2 v+\frac{1}{2}(2+v)\left(x^{2}+y^{2}-1\right)\right]\right], \\
v^{\prime}=v(q+1) .
\end{gathered}
$$

Here, primes denote differentiation with respect to $\tau$, and $q=$ $-\ddot{a} a / \dot{a}^{2}$ stands for the deceleration parameter

$$
\begin{aligned}
q+1= & \frac{3}{2}\left(x^{2}+\left[1+w_{\text {curv }}\left(1-x^{2}\right)\right] y^{2}\right) \\
& \times\left(4 x^{2}+8 y^{2}-x^{2} y^{2}-\frac{3}{2} y^{4}+\frac{1}{4} v y^{4}-\frac{9}{4} v y^{2}\right. \\
& \left.-\frac{1}{2} v x^{2}-\frac{19}{2} v-5\right)^{-1} .
\end{aligned}
$$

To study cosmological evolution in the dynamical system approach, it is necessary to find fixed (or critical) points of the model that are denoted by $\left(x^{*}, y^{*}, v^{*}\right)$. These points are achieved by fulfillment of the following condition:

$$
g^{i}\left(x^{*}, y^{*}, v^{*}\right)=0
$$

where

$$
x^{\prime i}=g^{i}\left(x^{*}, y^{*}, v^{*}\right) \text {, }
$$

where in Table $1 x_{B}$ and $y_{B}$ are as follows:

$$
x_{B}=\sqrt{x_{B}^{2}\left(w_{\mathrm{curv}}-1\right)+\left(1+w_{\mathrm{curv}}\right)},
$$

A part of dynamical system analysis of this model is summarized in Table 1.

The critical points $A$ and $B$ demonstrate the earlytime, matter-dominated epoch which leads to a positive deceleration parameter. Points $C$ and $D$ which are phases with vanishing matter character, that is, $\Omega_{m}=0$, can explain the positively accelerated phase of the universe expansion for $w_{\text {curv }} \geq-6.88$. Critical curve $E$ also demonstrates a positively accelerated phase for all values of the equation of state parameter of the curvature fluid. Critical curve $F$, which exists only for the case $w_{\text {curv }}=-1$, is a de Sitter phase in this model. Existence of a stable de Sitter point and an unstable matter-dominated phase (in addition to radiationdominated era) in the universe expansion history is required for cosmological viability of any cosmological model. In order to investigate the stability of these points, one can obtain the eigenvalues of these points separately. Based on Table 2, in order for point $A$ to be an unstable point, it is necessary to have $w_{\text {curv }}<1 / 4$. Therefore, the point $A$ as a saddle point agrees with what we have shown in Figure 7. Now, the stability of the positively accelerated phases of the model depends on whether the curvature fluid plays the role of a quintessence 
TABLE 1: Location and deceleration parameter of the critical points. The location of point $B$ and the deceleration factor of points $C$ and $D$ are dependent on the equation of state parameter of the perfect fluid, $w_{\text {curv }}$. The critical curve $F$ exists just for $w_{\text {curv }}=-1$.

\begin{tabular}{lcccc}
\hline Name & $x$ & $y$ & $v$ & $q$ \\
\hline$A$ & $\sqrt{15 / 3}$ & 0 & 0 & $1 / 2$ \\
$B$ & $x_{B}$ & $y_{B}$ & 0 & $-\left(1.17+0.17 w_{\text {curv }}\right)$ \\
$C$ & 0 & $1 / 2$ & 0 & $-\left(1.13+0.13 w_{\text {curv }}\right)$ \\
$D$ & 0 & $7 / 2$ & 0 & -1 \\
$F$ & 0 & 0 & $v^{*}$ & -1 \\
\hline
\end{tabular}

TABLE 2: Eigenvalues and the stability regions of the critical points. $\varsigma\left(w_{\text {curv }}\right), \xi\left(w_{\text {curv }}\right)$, and $\zeta\left(w_{\text {curv }}\right)$ are complicated functions of $w_{\text {curv }}$. The critical curve $F$ for which $w_{\text {curv }}=-1$ is a stable de Sitter phase.

\begin{tabular}{lcr}
\hline Name & Eigenvalue & Stable region \\
\hline$A$ & $-9,-1 / 2,1-4 w_{\text {curv }}$ & $w_{\text {curv }}>1 / 4$ \\
$B$ & $\varsigma\left(w_{\text {curv }}\right), \xi\left(w_{\text {curv }}\right), \zeta\left(w_{\text {curv }}\right)$ & - \\
$C$ & $-\left(1+0.1 w_{\text {curv }}\right),-\left(1+w_{\text {curv }}\right),-\left(2+0.17 w_{\text {curv }}\right)$ & $w_{\text {curv }}>-1$ \\
$D$ & $-\left(1+0.1 w_{\text {curv }}\right),-\left(1+w_{\text {curv }}\right),-\left(2+0.13 w_{\text {curv }}\right)$ & $w_{\text {curv }}>-1$ \\
$E$ & $-3 / 2,-3 / 2\left(1+w_{\text {curv }}\right),-2$ & $w_{\text {curv }}>-1$ \\
$F$ & $-3 / 2,0,-2$ & $w_{\text {curv }}=-1$ \\
\hline
\end{tabular}

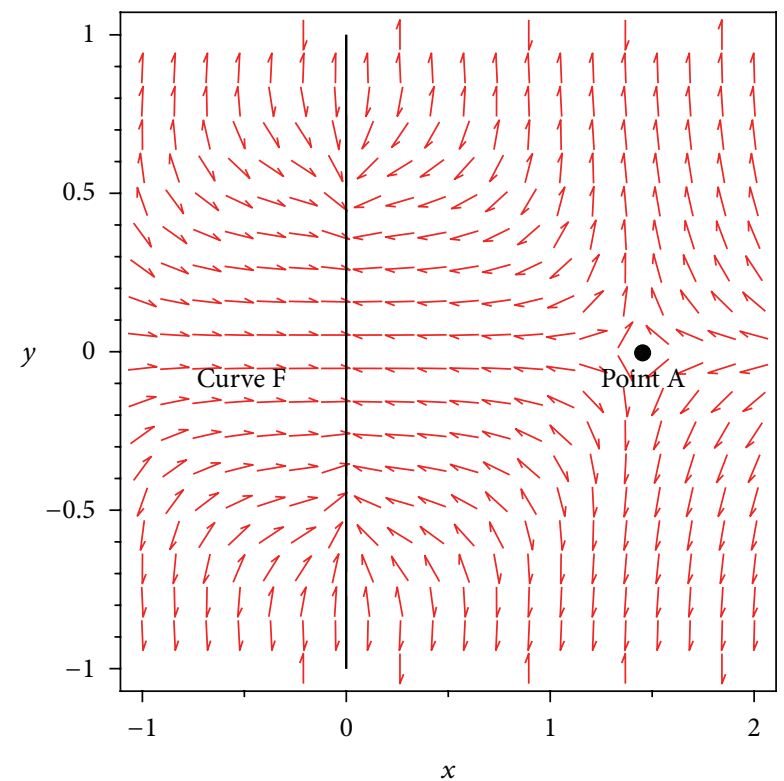

FIGURE 7: The phase subspace $x-y$ that the curvature fluid plays the role of a cosmological constant. This model is cosmologically viable since there are an unstable matter-dominated point and a stable de Sitter phase.

scalar field or not. Points $C, D$, and $E$ of Table 2 are stable phases of this model if $w_{\text {curv }}>-1$, whereas if the curvature fluid plays the role of a cosmological constant, the point $F$ will be a stable de Sitter phase. We note that, generally, if a nonlinear system has a critical curve, the Jacobian matrix of the linearized system at a critical point on the curve (line in our 2-dimensional subspace) has a zero eigenvalue with an associated eigenvector tangent to the critical curve at the chosen point. The stability of a specific critical point on the curve can be determined by the nonzero eigenvalues, because near this critical point there are essentially no dynamics along the critical curve [102]. We have plotted the phase space of this model in subspace $x-y$ with $w_{\text {curv }}=-1$. As we see, point $A$ is a saddle point, and curve $F$ is a stable de Sitter curve.

\section{Confrontation with Recent Observational Data}

In this section, we use the combined data from Planck + WMAP + high L+ lensing + BAO [103] to confront our model with recent observation. In this way, we obtain some constraints on the model parameters, especially, the GaussBonnet curvature contribution. For this purpose, we consider the relation between $\Omega_{\text {curv }}$ and $\Omega_{m}$ in the background of the mentioned observational data. We suppose that $\Omega_{\text {curv }}$ plays the role of dark energy in this setup. Figure 8 shows the result of our numerical study. In this model, with $\Omega_{r_{c}} \sim 10^{-4}$ (see [104], e.g.,), $\Omega_{\alpha}$ is constrained as follows:

$$
\begin{array}{lc}
w_{\text {curv }}=-0.5, & 0.008<\Omega_{\alpha}<0.011, \\
w_{\text {curv }}=-0.92, & 0.01<\Omega_{\alpha}<0.055, \\
w_{\text {curv }}=-1.05, & 0.012<\Omega_{\alpha}<0.073 .
\end{array}
$$

On the other hand, if we consider the $\Omega_{\text {eff }}$ defined as $\Omega_{\text {eff }}=\left(\kappa_{4}^{2} / 3 H_{0}^{2}\right) \rho_{\text {eff }}$ as our main parameter, the result will be as shown in Figure 9. In this case, we have the following constraint on $\Omega_{\alpha}$ :

$$
\begin{array}{lr}
\Omega_{\text {curv }}=0.7, & 0.0043<\Omega_{\alpha}<0.036, \\
\Omega_{\text {curv }}=0.5, & 0.0048<\Omega_{\alpha}<0.0081 .
\end{array}
$$




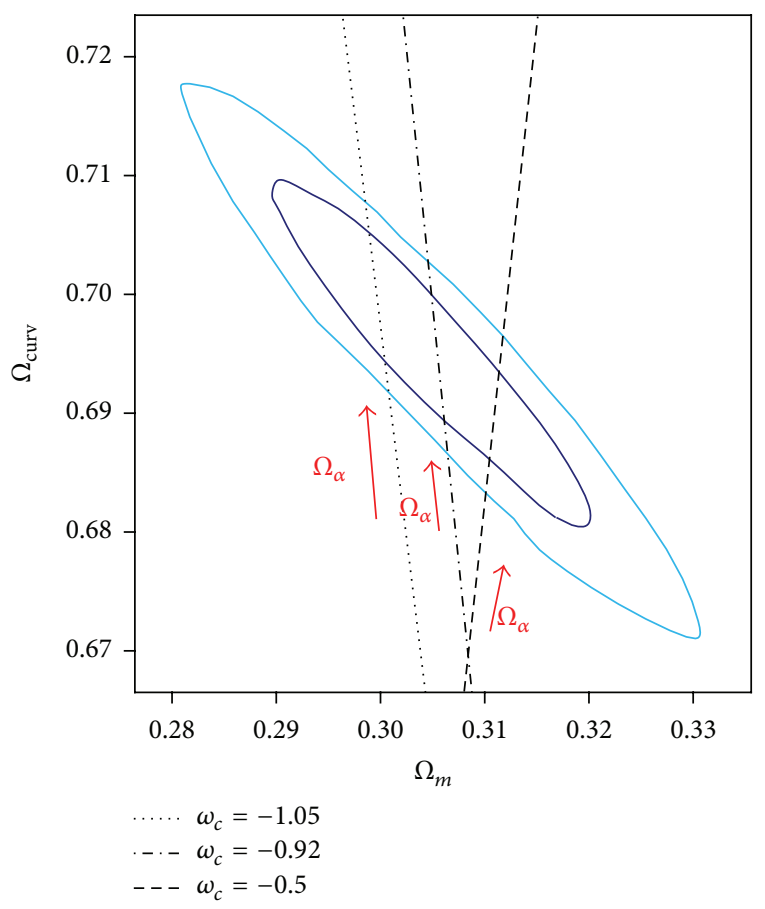

FIgURE 8: $\Omega_{\text {curv }}$ versus $\Omega_{m}$ in the background of Planck + WMAP + high $\mathrm{L}+$ lensing $+\mathrm{BAO}$ joint data.

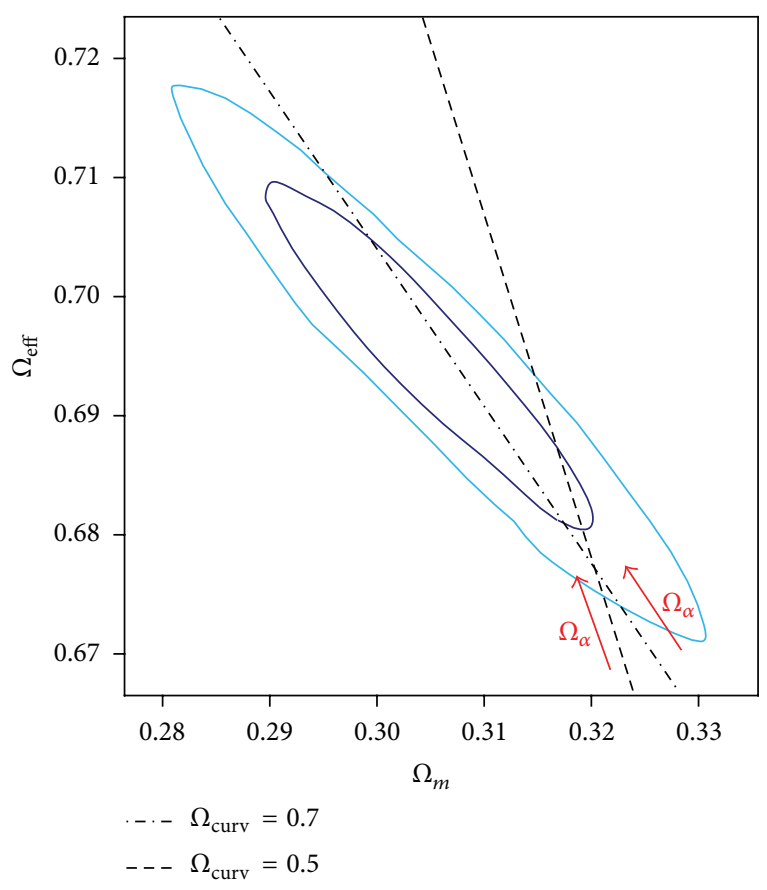

FIgURE 9: $\Omega_{\text {eff }}$ versus $\Omega_{m}$ in the background of Planck + WMAP + high $\mathrm{L}+$ lensing $+\mathrm{BAO}$ joint data.

\section{Summary and Conclusion}

In this paper, we have constructed a DGP-inspired braneworld scenario where induced gravity on the brane is modified in the spirit of $f(R)$ gravity, and higher-order curvature effects are taken into account by incorporation of the Gauss-Bonnet term in the bulk action. It is well known that the normal branch of the DGP braneworld scenario, which is not self-accelerating, has the potential to realize phantom-like behavior without introducing any phantom fields neither on the brane nor in the bulk. Our motivation here to study this extension of the DGP setup is to explore possible influences of the curvature corrections, especially, the modified induced gravity, on the cosmological dynamics of the normal branch of the DGP setup. In this regard, cosmological dynamics of this scenario as an alternative for dark energy proposal is studied, and the effects of the curvature corrections on the phantom-like dynamics of the model are investigated. The complete analysis of the generalized Friedmann equation needs a cosmographic viewpoint to $f(R)$ gravity, but here we have tried to find a special solution of this generalized equation via the discriminant function method. In our framework, effective energy density attributed to the curvature plays the role of effective dark energy density. In other words, we defined a curvature fluid with varying equation of state parameter that incorporates in the definition of effective dark energy density. The equation of state parameter of this curvature fluid is evolving, and the effective dark energy equation of state parameter has transition from quintessence to the phantom phase in a nonsmooth manner. We have considered a cosmologically viable (Hu-Sawicki) ansatz for $f(R)$ gravity on the brane to have more practical results. We have shown that this model mimics the phantom-like behavior on the normal branch of the scenario in some subspaces of the model parameter space without introduction of any phantom matter neither in the bulk nor on the brane. At the same time, the null energy condition is respected in the phantom-like phase of the model parameter space. There is no super-acceleration or big-rip singularity in this setup. Incorporation of the curvature effects both in the bulk (via the Gauss-Bonnet term) and on the brane (via modified induced gravity) results in the facility that curvature fluid plays the role of dark energy component. On the other hand, this extension allows the model to mimic the phantom-like prescription in relatively wider range of redshifts in comparison to the case that induced gravity is not modified. This effective phantomlike behavior permits us to study cosmological dynamics of this setup from a dynamical system viewpoint. This analysis has been performed with details, and its consequences are explained. The detailed dynamical system analysis of this setup is more involved relative to the case that there are no curvature effects. We have shown that, with suitable condition on equation of state parameter of curvature fluid, there are an unstable matter era and a stable de Sitter phase in this scenario leading to the conclusion that this model is cosmologically viable. We have constrained our model based on the recent observational data from joint Planck + WMAP + high L+ lensing + BAO data sets. In this way, some constraints on Gauss-Bonnet coupling contribution are presented. We note that no big-rip singularity is present in this model since the Gauss-Bonnet contribution to this model is essentially a stringy, quantum gravity effect that prevents the big-rip singularity (see, e.g., [105] for details). 


\section{Acknowledgment}

The work of K. Nozari has been supported financially by the Center for Excellence in Astronomy and Astrophysics (CEAAI-RIAAM), Maragha, Iran.

\section{References}

[1] S. Perlmutter, G. Aldering, and G. Goldhaber, "Measurements of $\Omega$ and $\Lambda$ from 42 high-redshift supernovae," The Astrophysical Journal, vol. 517, p. 565, 1999.

[2] A. G. Riess, A. V. Filippenko, P. Challis et al., "Observational evidence from supernovae for an accelerating universe and a cosmological constant," The Astronomical Journal, vol. 116, p. 1006, 1998.

[3] A. D. Miller, R. Caldwell, and M. J. Devlin, "A measurement of the angular power spectrum of the cosmic microwave background from $l=100$ to 400," The Astrophysical Journal Letters, vol. 524, p. L1, 1999.

[4] P. de Bernardis, P. A. R. Ade, J. J. Bock et al., "A flat Universe from high-resolution maps of the cosmic microwave background radiation," Nature, vol. 404, p. 955, 2000.

[5] S. Hanany, P. Ade, A. Balbi et al., "MAXIMA-1: a measurement of the cosmic microwave background anisotropy on angular scales of 10-5 ", The Astrophysical Journal Letters, vol. 545, p. L5, 2000.

[6] D. N. Spergel, L. Verde, H. V. Peiris et al., "First-year Wilkinson Microwave Anisotropy Probe (WMAP) observations: determination of cosmological parameters," The Astrophysical Journal, vol. 148, p. 175, 2003.

[7] L. Page, M. R. Nolta, C. Barnes et al., "First-Year Wilkinson Microwave Anisotropy Probe (WMAP) Observations: interpretation of the TT and TE angular power spectrum peaks," The Astrophysical Journal, vol. 148, p. 233, 2003.

[8] G. Hinshaw, J. L. Weiland, R. S. Hill et al., "Five-year Wilkinson microwave anisotropy probe observations: data processing, sky maps, and basic results," The Astrophysical Journal, vol. 180, p. 225, 2009.

[9] D. N. Spergel, R. Bean, O. Doré et al., "Three-year Wilkinson Microwave Anisotropy Probe (WMAP) observations: implications for cosmology," The Astrophysical Journal, vol. 170, p. 377, 2007.

[10] G. Hinshaw, M. R. Nolta, C. L. Bennett et al., "Three-Year Wilkinson Microwave Anisotropy Probe (WMAP) observations: temperature analysis," The Astrophysical Journal, vol. 170, p. 288, 2007.

[11] L. Page, G. Hinshaw, E. Komatsu et al., “Three-year Wilkinson Microwave Anisotropy Probe (WMAP) observations: polarization analysis," The Astrophysical Journal, vol. 170, p. 335, 2007.

[12] A. G. Reiss, L.-G. Strolger, J. Tonry et al., "Type Ia Supernova Discoveries at $z>1$ from the Hubble Space Telescope: evidence for past deceleration and constraints on dark energy evolution," The Astrophysical Journal, vol. 607, p. 665, 2004.

[13] S. W. Allen, R. W. Schmidt2, H. Ebeling3, A. C. Fabian1, and L. Van Speybroeck, "Constraints on dark energy from Chandra observations of the largest relaxed galaxy clusters," Monthly Notices of the Royal Astronomical Society, vol. 353, p. 457, 2004.

[14] E. Komatsu, J. Dunkley, and M. R. Nolta, "Five-year wilkinson microwave anisotropy probe observations: cosmological interpretation," The Astrophysical Journal, vol. 180, p. 330, 2009.
[15] E. J. Copeland, M. Sami, and S. Tsujikawa, "Dynamics of dark energy," International Journal of Modern Physics D, vol. 15, no. 11, pp. 1753-1935, 2006.

[16] H. Wei and R. G. Cai, "Interacting vectorlike dark energy, the first and second cosmological coincidence problems," Physical Review D, vol. 73, Article ID 083002, 10 pages, 2006.

[17] A. Vikman, "Can dark energy evolve to the phantom?" Physical Review D, vol. 71, Article ID 023515, 14 pages, 2005.

[18] S. Nojiri and S. D. Odintsov, "Unifying phantom inflation with late-time acceleration: scalar phantom-non-phantom transition model and generalized holographic dark energy," General Relativity and Gravitation, vol. 38, pp. 1285-1304, 2006.

[19] S. Nojiri, S. D. Odintsov, and S. Tsujikawa, "Properties of singularities in the (phantom) dark energy universe," Physical Review D, vol. 71, Article ID 063004, 16 pages, 2005.

[20] E. Elizalde, S. Nojiri, S. D. Odintsov, and P. Wang, "Dark energy: vacuum fluctuations, the effective phantom phase, and holography," Physical Review D, vol. 71, Article ID 103504, 8 pages, 2005.

[21] U. Alam, V. Sahni, and A. A. Starobinsky, "The case for dynamical dark energy revisited," Journal of Cosmology and Astroparticle Physics, vol. 2004, 06, article 008, 2004.

[22] S. Nesseris and L. Perivolaropoulos, "Comparison of the legacy and gold type Ia supernovae dataset constraints on dark energy models," Physical Review D, vol. 72, Article ID 123519, 8 pages, 2005.

[23] S. D. Sadatian and K. Nozari, "Crossing of the phantom divided barrier with Lorentz invariance violating fields," Europhysics Letters, vol. 82, Article ID 49001, 2008.

[24] S. D. Sadatian and K. Nozari, "A Lorentz invariance violating cosmology on the DGP brane," Europhysics Letters, vol. 2009, 01, article 005, 2009.

[25] R. J. Scherrer and A. A. Sen, "Phantom dark energy models with a nearly flat potential," Physical Review D, vol. 78, Article ID 067303, 4 pages, 2008.

[26] F. Briscese, E. Elizalde, S. Nojiri, and S. D. Odintsov, "Phantom scalar dark energy as modified gravity: understanding the origin of the big rip singularity," Physics Letters B, vol. 646, no. 2-3, pp. 105-111, 2007.

[27] Y. F. Cai, T. Qiu, X. Zhang, Y.-S. Piao, and M. Li, "Bouncing universe with Quintom matter," Journal of High Energy Physics, vol. 2007, article 071, 2007.

[28] M. R. Setare and E. N. Saridakis, "Coupled oscillators as models of quintom dark energy," Physics Letters B, vol. 668, no. 3, pp. 177-181, 2008.

[29] M. Sami, "A primer on problems and prospects of dark energy," Current Science, vol. 97, p. 887, 2009.

[30] S. Nesseris and L. Perivolaropoulos, "Crossing the phantom divide: theoretical implications and observational status," Journal of Cosmology and Astroparticle Physics, vol. 2007, article 018, 2007.

[31] K. Bamba, S. Capozziello, S. Nojiri, and S. D. Odintsov, "Dark energy cosmology: the equivalent description via different theoretical models and cosmography tests," Astrophysics and Space Science, vol. 342, pp. 155-228, 2012.

[32] S. Nojiri and S. D. Odintsov, "Introduction to modified gravity and gravitational alternative for dark energy," International Journal of Geometric Methods in Modern Physics, vol. 4, no. 1, pp. 115-145, 2007.

[33] S. Nojiri and S. D. Odintsov, "Can quantum-corrected BTZ black hole anti-evaporate?” Modern Physics Letters A, vol. 13, no. 33, pp. 2695-2704, 1998. 
[34] T. P. Sotiriou and V. Faraoni, " $f(R)$ theories of gravity," Reviews of Modern Physics, vol. 82, no. 1, pp. 451-497, 2010.

[35] S. Capozziello and M. Francaviglia, "Extended theories of gravity and their cosmological and astrophysical applications," General Relativity and Gravitation, vol. 40, no. 2-3, pp. 357-420, 2008.

[36] R. Durrer and R. Maartens, "Dark energy and dark gravity: theory overview," in Dark Energy: Observational \& Theoretical Approaches, pp. 48-91, 2010.

[37] K. Bamba, S. Nojiri, and S. D. Odintsov, "Future of the universe in modified gravitational theories: approaching to the finitetime future singularity," Journal of Cosmology and Astroparticle Physics, vol. 2008, article 045, 2008.

[38] S. Jhingan, S. Nojiri, S. D. Odintsov, M. Sami, I. Thongkool, and S. Zerbini, "Phantom and non-phantom dark energy: the cosmological relevance of non-locally corrected gravity," Physics Letters B, vol. 663, no. 5, pp. 424-428, 2008.

[39] R. Durrer and R. Maartens, "Dark energy and dark gravity: theory overview," General Relativity and Gravitation, vol. 40, no. 2-3, pp. 301-328, 2008.

[40] S. Capozziello and V. Salzano, "Cosmography and large scale structure by $f(R)$ gravity: new results," Advances in Astronomy, vol. 2009, Article ID 217420, 34 pages, 2009.

[41] S. Capozziello, V. F. Cardone, and V. Salzano, "Cosmography of $f(R)$ gravity," Physical Review D, vol. 78, no. 6, Article ID 063504, 2008.

[42] S. Bellucci, S. Capozziello, M. De Laurentis, and V. Faraoni, "Position and frequency shifts induced by massive modes of the gravitational wave background in alternative gravity," Physical Review D, vol. 79, no. 10, Article ID 104004, 2009.

[43] S. Nojiri and S. D. Odintsov, "Unified cosmic history in modified gravity: from $f(R)$ theory to Lorentz non-invariant models," Physics Reports, vol. 505, pp. 59-144, 2011.

[44] A. de Felice and S. Tsujikawa, " $f(R)$ theories," Living Reviews in Relativity, vol. 13, no. 3, pp. 1-161, 2010.

[45] S. Tsujikawa, "Modified gravity models of dark energy," Lecture Notes in Physics, vol. 800, pp. 99-145, 2010.

[46] G. Dvali, G. Gabadadze, and M. Porrati, " $4 \mathrm{D}$ gravity on a brane in 5D Minkowski space," Physics Letters B, vol. 485, no. 1-3, pp. 208-214, 2000.

[47] G. Dvali and G. Gabadadze, "Gravity on a brane in infinitevolume extra space," Physical Review D, vol. 63, no. 6, Article ID 065007, 2001.

[48] G. Dvali, G. Gabadadze, M. Kolanović, and F. Nitti, "Scales of gravity," Physical Review D, vol. 65, no. 2, Article ID 024031, 2002.

[49] C. Deffayet, "Cosmology on a brane in Minkowski bulk," Physics Letters B, vol. 502, pp. 199-208, 2001.

[50] C. Deffayet, G. Dvali, and G. Gabadadze, "Accelerated universe from gravity leaking to extra dimensions," Physical Review D, vol. 65, no. 4, Article ID 044023, 2002.

[51] C. Deffayet, S. J. Landau, J. Raux, M. Zaldarriaga, and P. Astier, "Supernovae, $\mathrm{CMB}$, and gravitational leakage into extra dimensions," Physical Review D, vol. 66, no. 2, Article ID 024019, 2002.

[52] K. Koyama, "Ghosts in the self-accelerating universe," Classical and Quantum Gravity, vol. 24, no. 24, pp. R231-R253, 2007.

[53] C. de Rham and A. J. Tolley, "Mimicking $\Lambda$ with a spintwo ghost condensate," Journal of Cosmology and Astroparticle Physics, vol. 2006, 07, article 004, 2006.
[54] V. Sahni and Y. Shtanov, "Braneworld models of dark energy," Journal of Cosmology and Astroparticle Physics, no. 11, article 014, 2003.

[55] A. Lue and G. D. Starkman, "How a brane cosmological constant can trick us into thinking that $w<-1$," Physical Review D, vol. 70, no. 10, Article ID 101501, 2004.

[56] V. Sahni, "Cosmological Surprises from Braneworld models of Dark Energy," in Proceedings of the 14th Workshop on General relativity and Gravitation (JGRG '04), pp. 95-115, Kyoto University, Kyoto, Japan, 2004.

[57] L. P. Chimento, R. Lazkoz, R. Maartens, and I. Quiros, "Crossing the phantom divide without phantom matter," Journal of Cosmology and Astroparticle Physics, vol. 2006, 09, article 004, 2006.

[58] R. Lazkoz, R. Maartens, and E. Majerotto, "Observational constraints on phantomlike braneworld cosmologies," Physical Review D, vol. 74, no. 8, Article ID 083510, 2006.

[59] R. Maartens and E. Majerotto, "Observational constraints on self-accelerating cosmology," Physical Review D, vol. 74, Article ID 023004, 6 pages, 2006.

[60] M. Bouhmadi-Lopez, "Phantom-like behaviour in dilatonic brane-world scenario with induced gravity," Nuclear Physics B, vol. 797, pp. 78-92, 2008.

[61] K. Nozari and M. Pourghassemi, "Crossing the phantom divide line in a Dvali-Gabadadze-Porrati-inspired $f(R, \phi)$ gravity," Journal of Cosmology and Astroparticle Physics, vol. 2008, article 044, 2008.

[62] K. Nozari, "DGP cosmology with a non-minimally coupled scalar field on the brane," Journal of Cosmology and Astroparticle Physics, vol. 2007, 09, article 003, 2007.

[63] K. Atazadeh and H. R. Sepangi, "Modified gravity inspired DGP brane cosmology," Physics Letters B, vol. 643, no. 2, pp. 76-80, 2006.

[64] K. Atazadeh and H. R. Sepangi, "Curvature corrections in DGP brane cosmology," Journal of Cosmology and Astroparticle Physics, no. 9, article 020, 2007.

[65] K. Atazadeh, M. Farhoudi, and H. R. Sepangi, "Accelerating universe in $f(R)$ brane gravity," Physics Letters B, vol. 660, no. 4, pp. 275-281, 2008.

[66] J. Saavedra and Y. Vasquez, "Effective gravitational equations on brane world with induced gravity described by $f(R)$ term," Journal of Cosmology and Astroparticle Physics, vol. 2009, 04, article 013, 2009.

[67] M. Bouhmadi-Lopez, "Self-accelerating the normal DGP branch," Journal of Cosmology and Astroparticle Physics, vol. 2009, article 011, 2009.

[68] K. Nozari and F. Kiani, "Dynamical-screening and the phantom-like effects in a DGP-inspired $f(R, \phi)$ model," Journal of Cosmology and Astroparticle Physics, vol. 2009, article 010, 2009.

[69] P. S. Apostolopoulos, N. Brouzakis, N. Tetradis, and E. Tzavara, "Modified brane cosmologies with induced gravity, arbitrary matter content, and a Gauss-Bonnet term in the bulk," Physical Review D, vol. 76, no. 8, Article ID 084029, 2007.

[70] R.-G. Cai, H.-S. Zhang, and A. Wang, "Crossing $w=-1$ in Gauss-Bonnet brane world with induced gravity," Communications in Theoretical Physics, vol. 44, article 948, 2005.

[71] E. Browne and T. Browne, "Conversations on the mountain range border," Architectural Research Quarterly, no. 71, pp. 6871, 2009. 
[72] R. A. Brown, R. Maartens, E. Papantonopoulos, and V. Zamarias, "A late-accelerating universe with no dark energyand a finite-temperature big bang," Journal of Cosmology and Astroparticle Physics, vol. 2005, article 008, 2005.

[73] S. Nojiri, S. D. Odintsov, and P. V. Tretyakov, "Dark energy from modified $f(R)$-scalar-Gauss-Bonnet gravity," Physics Letters $B$, vol. 651, no. 2-3, pp. 224-231, 2007.

[74] G. Cognola, E. Elizalde, S. Nojiri, S. D. Odintsov, and S. Zerbini, "Dark energy in modified Gauss-Bonnet gravity: latetime acceleration and the hierarchy problem," Physical Review D, vol. 73, no. 8, Article ID 084007, pp. 1-16, 2006.

[75] S. Nojiri and S. D. Odintsov, "Modified Gauss-Bonnet theory as gravitational alternative for dark energy," Physics Letters B, vol. 631, no. 1-2, pp. 1-6, 2005.

[76] S. Nojiri, S. D. Odintsov, and M. Sasaki, "Gauss-Bonnet dark energy," Physical Review D, vol. 71, Article ID 123509, 7 pages, 2005.

[77] E. N. Saridakis, "Holographic dark energy in braneworld models with a Gauss-Bonnet term in the bulk. Interacting behavior and the $w=-1$ crossing," Physics Letters B, vol. 661, no. 5, pp. 335-341, 2008.

[78] M. Alimohammadi and A. Ghalee, "Remarks on generalized Gauss-Bonnet dark energy," Physical Review D, vol. 79, no. 6, Article ID 063006, 2009.

[79] K. Nozari, T. Azizi, and M. R. Setare, "Phantom-like behavior of a DGP-inspired Scalar-Gauss-Bonnet gravity," Journal of Cosmology and Astroparticle Physics, vol. 2009, article 022, 2009.

[80] K. Nozari and T. Azizi, "Phantom-like effects in an asymmetric brane embedding with induced gravity and the Gauss-Bonnet term in the bulk," Physica Scripta, vol. 83, Article ID 015001, 2011.

[81] K. Nozari and N. Rashidi, "Modified GBIG scenario as an alternative for dark energy," Journal of Cosmology and Astroparticle Physics, vol. 2009, article 014, 2009.

[82] K. Nozari and N. Rashidi, "A braneworld dark energy model with induced gravity and the Gauss-Bonnet effect," International Journal of Theoretical Physics, vol. 48, no. 10, pp. 28002817, 2009.

[83] K. Nozari and N. Alipour, "Phantom-like behavior with curvature effect and modified induced gravity," Europhysics Letters, vol. 87, Article ID 69001, 2009.

[84] K. Nozari, T. Azizi, and N. Alipour, "Observational constraints on Chaplygin cosmology in a braneworld scenario with induced gravity and curvature effect," Monthly Notices of the Royal Astronomical Society, vol. 412, no. 4, pp. 2125-2136, 2011.

[85] S. Nojiri, S. D. Odintsov, and S. Ogushi, "FriedmannRobertson-Walker brane cosmological equations from the fivedimensional bulk (A)DS black hole," International Journal of Modern Physics A, vol. 17, p. 4809, 2002.

[86] M. Bouhmadi-Lopez and P. Vargas Moniz, "Phantomlike behavior in a brane-world model with curvature effects," Physical Review D, vol. 78, Article ID 084019, 2008.

[87] M. Bouhmadi-Lopez and A. Ferrera, "Crossing the cosmological constant line in a dilatonic brane-world model with and without curvature corrections," Journal of Cosmology and Astroparticle Physics, vol. 2008, article 011, 2008.

[88] M. Bouhmadi-López and P. V. Moniz, "Late-time acceleration in a brane with curvature effects," in Proceedings of the Spanish Relativity Meeting, vol. 1122, pp. 201-204, September 2008.

[89] M. Bouhmadi-Lopez, Y. Tavakoli, and P. Vargas Moniz, "Appeasing the phantom menace?" Journal of Cosmology and Astroparticle Physics, vol. 2010, no. 4, article 016, 2010.
[90] M. Bouhmadi-López, A. Errahmani, and T. Ouali, "Cosmology of a holographic induced gravity model with curvature effects," Physical Review D, vol. 84, no. 8, Article ID 083508, 2011.

[91] K. Nozari and F. Kiani, "On the cosmological viability of the Hu-Sawicki type modified induced gravity," Physics Letters B, vol. 703, pp. 395-401, 2011.

[92] K. Nozari and B. Fazlpour, "Gauss-Bonnet cosmology with induced gravity and a non-minimally coupled scalar field on the brane," Journal of Cosmology and Astroparticle Physics, vol. 2008, no. 6, article 032, 2008.

[93] B. Gumjudpai, "Brane-cosmology dynamics with induced gravity," General Relativity and Gravitation, vol. 36, no. 4, pp. 747766, 2004.

[94] M. A. Luty, M. Porrati, and R. Rattazzi, "Strong interactions and stability in the DGP model," Journal of High Energy Physics, vol. 2003, no. 9, article 029, 2003.

[95] M. Bouhmadi-Lopez, S. Capozziello, and V. F. Cardone, "Cosmography of $f(R)$-brane cosmology," Physical Review D, vol. 82, Article ID 103526, 9 pages, 2010.

[96] W. Hu and I. Sawicki, "Models of $f(R)$ cosmic acceleration that evade solar-system tests," Physical Review D, vol. 76, Article ID 064004, 2007.

[97] G. F. R. Ellis and M. S. Madsen, "Exact scalar field cosmologies," Classical and Quantum Gravity, vol. 8, no. 4, pp. 667-676, 1991.

[98] R. Lazkoz and G. Leon, "Quintom cosmologies admitting either tracking or phantom attractors," Physics Letters B, vol. 638, pp. 303-309, 2006.

[99] C. G. Boehmer, G. Caldera-Cabral, R. Lazkoz, and R. Maartens, "Dynamics of dark energy with a coupling to dark matter," Physical Review D, vol. 78, Article ID 023505, 8 pages, 2008.

[100] K. Nozari and F. Kiani, "Cosmological dynamics with modified induced gravity on the normal DGP branch," Physics Letters B, vol. 718, p. 727, 2013.

[101] L. A. Urena-Lopez and M. J. Reyes-Ibarra, "On the dynamics of a quadratic scalar field potential," International Journal of Modern Physics D, vol. 18, p. 621, 2009.

[102] S.-Y. Zhou, E. J. Copeland, and P. M. Saffin, "Cosmological constraints on $f(G)$ dark energy models," Journal of Cosmology and Astroparticle Physics, vol. 2009, article 009, 2009.

[103] H. Adeli and N.-T. Cheng, "Augmented Lagrangian genetic algorithm for structural optimization," Journal of Aerospace Engineering, vol. 7, no. 1, pp. 104-118, 1994.

[104] T. Azizi, M. S. Movahed, and K. Nozari, "Observational constraints on the normal branch of a warped DGP cosmology," New Astronomy, vol. 17, no. 4, pp. 424-432, 2012.

[105] E. Elizalde, S. Nojiri, and S. D. Odintsov, "Late-time cosmology in (phantom) scalar-tensor theory: dark energy and the cosmic speed-up," Physical Review D, vol. 70, Article ID 043539, 2004. 

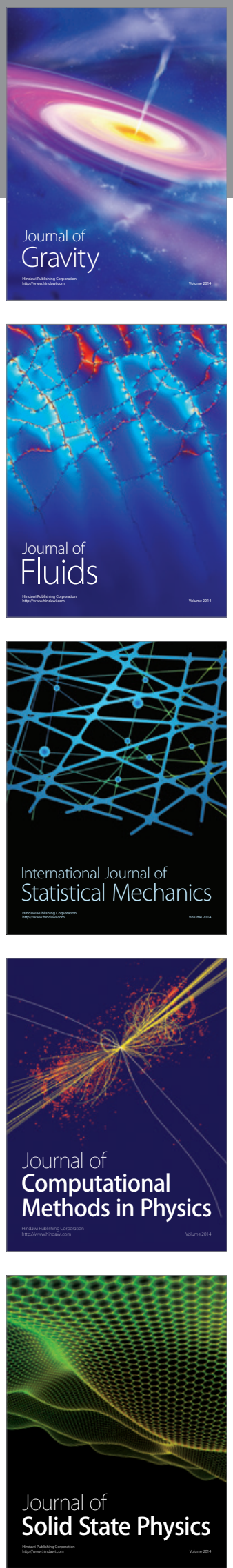

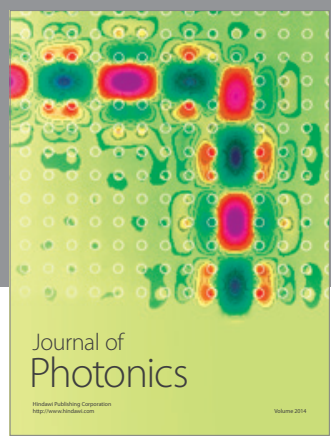

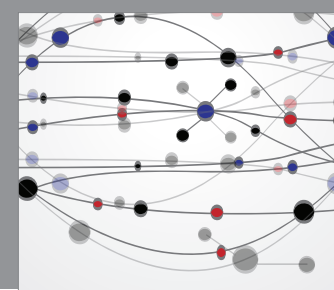

The Scientific World Journal

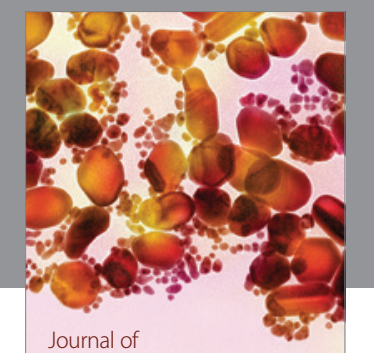

Soft Matter
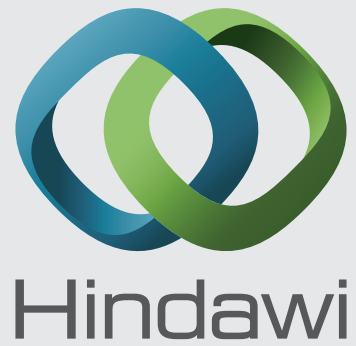

Submit your manuscripts at

http://www.hindawi.com
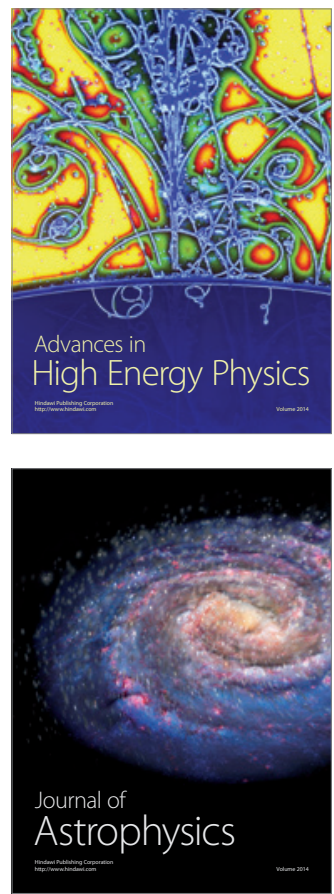
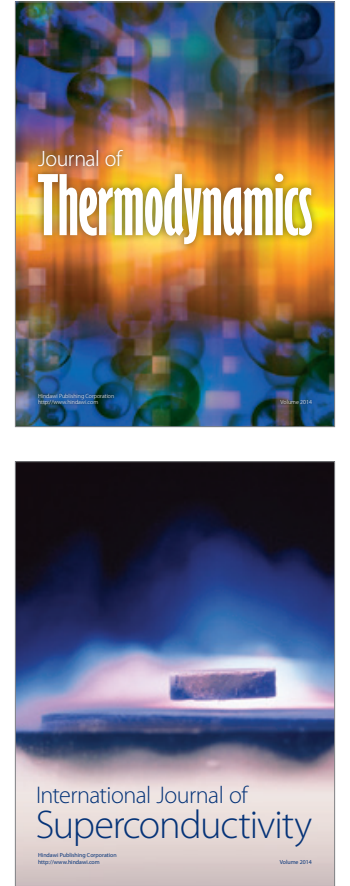
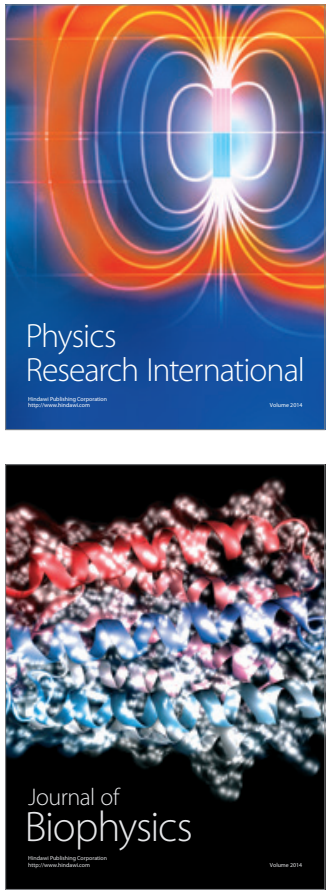
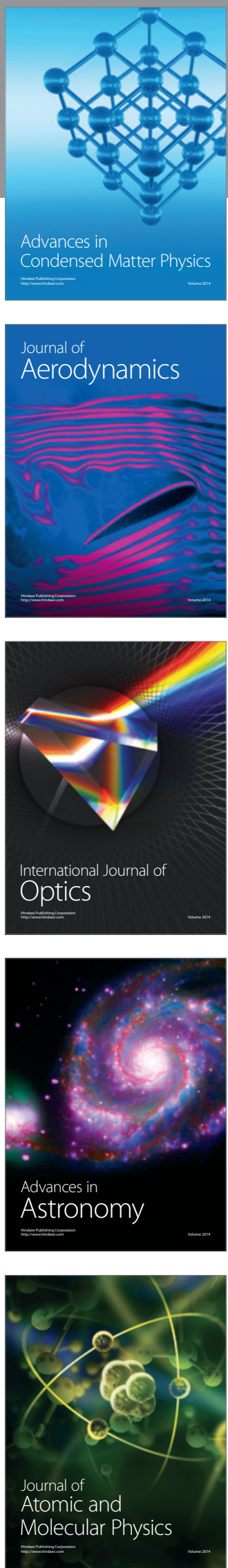